\title{
Granulocyte colony-stimulating factor treatment ameliorates lupus nephritis through the expansion of regulatory $T$ cells
}

Ji-Jing Yan $^{1 \dagger}$, Enkthuya Jambaldorj ${ }^{1 \dagger}$, Jae-Ghi Lee ${ }^{1}$, Joon Young Jang ${ }^{1}$, Jung Min Shim ${ }^{1}$, Miyeun Han², Tai Yeon Koo ${ }^{3}$, Curie $\mathrm{Ahn}^{2}$ and Jaeseok Yang ${ }^{1,3,4^{*}}$

\begin{abstract}
Background: Granulocyte colony-stimulating factor (G-CSF) can induce regulatory T cells (Tregs) as well as myeloidderived suppressor cells (MDSCs). Despite the immune modulatory effects of G-CSF, results of G-CSF treatment in systemic lupus erythematosus are still controversial. We therefore investigated whether G-CSF can ameliorate lupus nephritis and studied the underlying mechanisms.

Methods: NZB/W F1 female mice were treated with G-CSF or phosphate-buffered saline for 5 consecutive days every week from 24 weeks of age, and were analyzed at 36 weeks of age.

Results: G-CSF treatment decreased proteinuria and serum anti-dsDNA, increased serum complement component 3 (C3), and attenuated renal tissue injury including deposition of IgG and C3. G-CSF treatment also decreased serum levels of BUN and creatinine, and ultimately decreased mortality of NZB/W F1 mice. G-CSF treatment induced expansion of $\mathrm{CD}^{+}{ }^{+} \mathrm{CD} 25^{+} \mathrm{Foxp}^{+}$Tregs, with decreased renal infiltration of $\mathrm{T}$ cells, B cells, inflammatory granulocytes and monocytes in both kidneys and spleen. G-CSF treatment also decreased expression levels of MCP-1, IL-6, IL-2, and IL-10 in renal tissues as well as serum levels of MCP-1, IL-6, TNF-a, IL-10, and IL-17. When Tregs were depleted by PC61 treatment, G-CSF-mediated protective effects on lupus nephritis were abrogated.

Conclusions: G-CSF treatment ameliorated lupus nephritis through the preferential expansion of $C D 4^{+} \mathrm{CD} 25^{+}$Foxp3 + Tregs. Therefore, G-CSF has a therapeutic potential for lupus nephritis.
\end{abstract}

Keywords: Granulocyte colony-stimulating factor, Lupus nephritis, Regulatory $T$ cells

\section{Background}

Systemic lupus erythematosus (SLE) is an autoimmune disease in which altered $\mathrm{T}$ cell function and polyclonal B cell activation followed by autoantibody production including anti-double stranded DNA antibodies (anti-dsDNA), thus, leading to immune complex deposition in multiple organs, particularly in the kidney. These depositions drive local inflammatory responses that can lead to tissue damage and clinical disease $[1,2]$.

\footnotetext{
* Correspondence: jcyjs@dreamwiz.com

†Equal contributors

${ }^{1}$ Transplantation Research Institute, Seoul National University College of Medicine, Seoul 03080, Republic of Korea

${ }^{3}$ Transplantation Center, Seoul National University Hospital, Seoul 03080, Republic of Korea

Full list of author information is available at the end of the article
}

Granulocyte colony-stimulating factor (G-CSF) is a growth factor for neutrophils, that can accelerate neutrophil reconstitution after bone marrow suppression and activate effector functions of mature neutrophils [3]. G-CSF also stimulates proliferation, differentiation, and peripheral mobilization of hematopoietic stem cells [3]. Furthermore, G-CSF has been reported to modulate both $\mathrm{T}$ cell and innate immune responses. G-CSF can induce $\operatorname{Tr} 1$ cells in vitro, and mobilize $\mathrm{CD} 4{ }^{+} \mathrm{CD} 25$ ${ }^{+}$Foxp3 regulatory $\mathrm{T}$ cells (Tregs) $[4,5]$. In addition, GCSF can mobilize human tolerogenic dendritic cells and induce human semi-mature dendritic cells, and thereby induce type 1 regulatory $\mathrm{T}(\mathrm{Tr} 1)$ cells through interleukin (IL)-10 release [6, 7]. G-CSF suppresses production of IL-1 $\beta$, IL-12, interferon (IFN)- $\gamma$, and tumor necrosis factor (TNF)- $\alpha$, but increases serum levels of IFN- $\alpha$ 
and IL-10 [7, 8]. Recently, G-CSF was also reported to induce $\mathrm{CD} 11 \mathrm{~b}^{+} \mathrm{Gr}-1^{+}$myeloid-derived suppressor cells (MDSC), a subset of innate-like suppressor cells $[9,10]$. MDSCs are a heterogeneous population of immature myeloid cells that can suppress both $T$ cells and natural killer (NK) cells and thereby suppress autoimmunity as well as tumor immunity [10, 11]. MDSCs are divided into two subsets, granulocytic MDSCs $\left(\mathrm{CD} 11 \mathrm{~b}^{+}\right.$Ly6G $\left.{ }^{+} \mathrm{Ly} 6 \mathrm{C}^{\mathrm{low}}\right)$, and monocytic MDCSs $\left(\mathrm{CD} 11 \mathrm{~b}^{+} \mathrm{Ly}_{6 \mathrm{G}}{ }^{-}\right.$ Ly6C ${ }^{\text {high }}$ ) [10]. However, CD $11 b^{+}{ }^{+} y 6 G^{+}{\text {Ly } 6 C^{\text {low }}}$ and CD11 $b^{+}$Ly6G ${\text {Ly } 6 C^{\text {high }}}$ cells could be inflammatory granulocytes and inflammatory monocytes, respectively, because these populations share surface markers with MDSCs.

Despite the immunomodulatory effects of G-CSF, results of G-CSF treatment in SLE are still controversial. Low-dose G-CSF treatment accelerated lupus nephritis in MRL lymphoproliferation (MRL/lpr) strain mice and increased autoantibody production in B6.Sle1.Sle2.Sle3 spontaneous mouse model of lupus [12, 13]. In contrast, high-dose G-CSF treatment prevented lupus nephritis and delayed mortality [13]. In patients, G-CSF induced disease flares in both lupus nephritis and cutaneous lupus $[14,15]$. These controversial results require further study to confirm the role of G-CSF in lupus nephritis and to determine the involved mechanisms. In this study, we investigated whether G-CSF can ameliorate lupus nephritis in a NZB/W F1 mouse lupus model, and examined the related mechanisms.

\section{Methods}

\section{Animals and treatment regimens}

NZB/W F1 mice spontaneously develop a disease closely resembling human SLE [16]. Female NZB/W F1 mice were purchased from SLC Inc. (Hamamatsu, Japan) and housed under the pathogen-free conditions. The experimental group was injected subcutaneously with recombinant human G-CSF (Grasin, Kyowa Kirin, Korea) for 5 consecutive days every week from 24 weeks of age, at a dose of $250 \mu \mathrm{g} / \mathrm{kg} /$ day during 12 weeks. In low-dose experiments, $250 \mu \mathrm{g} / \mathrm{kg} /$ day of human G-CSF was administered 3 times a week for 12 weeks from 24 weeks of age. The control group received only phosphate-buffered saline (PBS) injection. For Treg depletion, depleting anti-CD25 antibodies (PC61, Bio X Cell, West Lebanon, NH, USA) were administered at a dose of $0.5 \mathrm{mg} 3$ times a week from 33 to 36 weeks.

\section{Measurement of proteinuria, renal function, anti-dsDNA, complement component 3 (C3), and cytokines}

Spot urine proteinuria was measured using protein reagent strips (URiSCAN; Yongdong Pharmaceutical Co., Seoul, Korea) once per week for 12 weeks. The measurement was semi-quantitative: $0=$ none or trace amount of proteinuria,
$1+, \quad 30-100 \mathrm{mg} / \mathrm{dL} ; 2+, \quad 100-300 \mathrm{mg} / \mathrm{dL} ; 3+, 300-$ $1000 \mathrm{mg} / \mathrm{dL} ; 4+, \geq 1000 \mathrm{mg} / \mathrm{dL}$. Urine albumin concentrations were measured using a mouse albumin ELISA kit (Alpco Diagnostics, Salem, NH, USA) and normalized to urine creatinine concentrations. Serum levels of BUN and creatinine were measured at 32 and 36 weeks using QuantiChrom urea and creatinine assay kits (BioAssay Systems, Hayward, CA, USA) [17]. Serum concentration of mouse anti-dsDNA and C3 were measured using ELISA kits (Alpha Diagnostic International, San Antonio, TX, USA; Abcam, Cambridge, MA, USA) at 36 weeks. Levels of monocyte chemoattractant protein-1 (MCP-1) and cytokines (IL-6, TNF- $\alpha$, IL-2, IFN- $\gamma$, IL-10, IL-4 and IL-17) were measured at 36 weeks in both serum and renal tissues using cytometric bead array kits (BD Biosciences, San Diego, CA, USA).

\section{Renal histologic analysis}

Paraffin sections of fixed kidneys were stained with Periodic Acid-Schiff's (PAS) stain kit, and evaluated according to described protocol $[18,19]$. Briefly, glomerular pathology was evaluated by assessing 20 glomerular crosssections (gcs) per kidney, and each glomerulus was scored on a semiquantitative scale: $0=$ normal $(35-40$ cells/glomerular cross-sections, gcs); $1=$ mild (glomeruli with few lesions, slight proliferative changes, mild hypercellularity, 41-50 cells/gcs); $2=$ moderate (glomeruli with moderate hypercellularity, 50-60 cells/gcs, segmental and/or diffuse proliferative changes, hyalinosis); 3 = severe (glomeruli with segmental or global sclerosis, and/or exhibiting severe hypercellularity ( $>60$ cells/gcs), necrosis, crescent formation). Interstitial/tubular pathology was assessed semiquantitatively on a scale of $0-3$ in 10 randomly selected high-power fields. We determined the largest and average number of infiltrates and damaged tubules and subsequently adjusted the grading system accordingly: 0 , normal; 1 , mild; 2 , moderate; 3 , severe. Perivascular cellular accumulation was determined semiquantitatively by scoring the number of cell layers surrounding the majority of vessel walls $(0$, none; $1,<5 ; 2,5-$ $10 ; 3, \geq 10$ cell layers).

Kidney cryostat sections were stained with goat antimouse IgG (Sigma-Aldrich) or rabbit anti-mouse C3 (Abcam) for $4 \mathrm{~h}$. Then, they were incubated at room temperature with Alexa Fluor 488 donkey anti-goat IgG or Alexa Fluor 568 donkey anti-rabbit IgG (Molecular Probe; Invitrogen USA) for $1 \mathrm{~h}$. Deposition of IgG and C3 within the peripheral glomerular capillary walls and the mesangium was measured as the mean fluorescence in 10 glomeruli per mouse. Scores were assigned based on the intensity of IgG/C3 deposition (0-3+), where 0 represents no deposition and 3 denotes intense deposition [20]. All histologic analysis was performed by two independent pathologists blinded to the treatment group. 
Flow cytometry

Renal leukocytes and splenocytes were pretreated with anti-mouse CD16/32 (clone 2.4G2) to block nonspecific Fc binding. Kidney leukocytes were identified by labeling cells with CD45 (30-F11). The fluorochrome-conjugated antibodies (BD Biosciences or eBioscience) were used for flow cytometric analysis: CD45 (30-F11), CD3 (1452C11), CD4 (RM4-5), CD8 (53-6.7), CD25 (ebioPC61), CD44 (IM7), CD69 (H1.2 F3), Foxp3 (ebio), CD11b (M1/70), Gr-1 (RB6-BC5, ebio), Ly6G (RB6-8c5), Ly6C (AL-21), CD19 (eBio1D3), CD138 (281-2), CXCR5 (SPRCL5), PD-1 (J43), NK1.1 (PK136). Appropriate
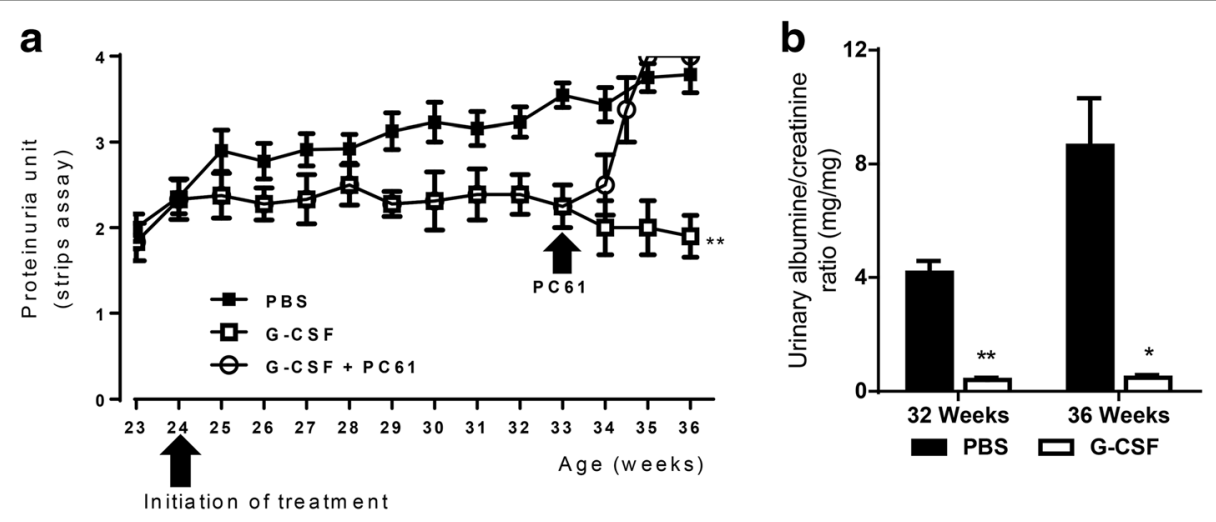

C

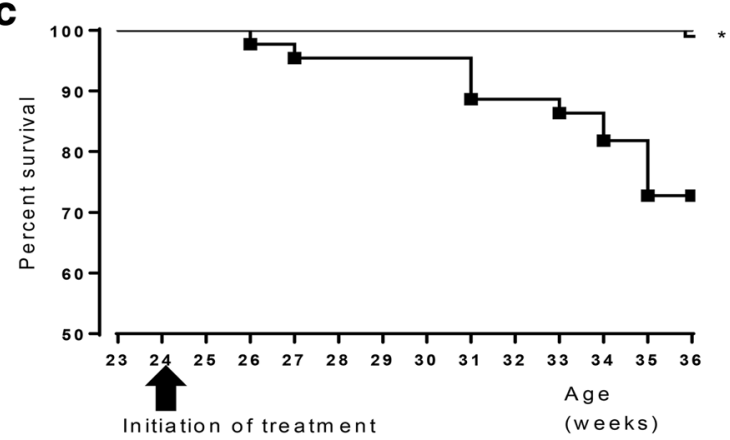

d

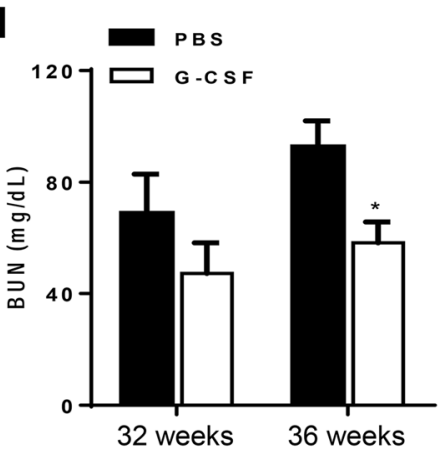

f

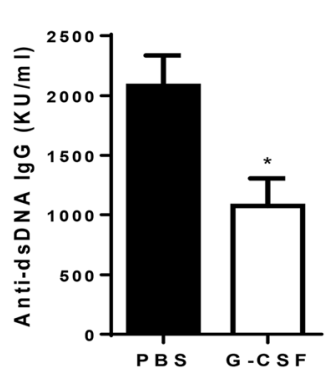

e

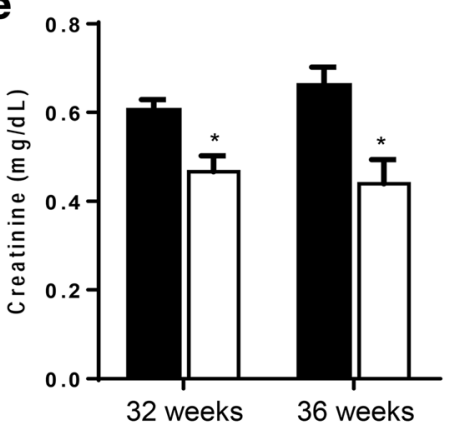

g

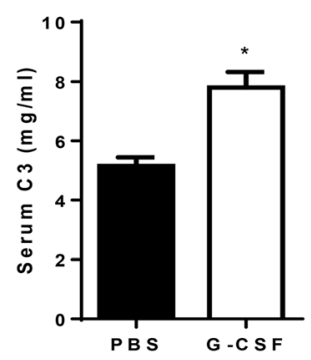

Fig. 1 (See legend on next page.) 


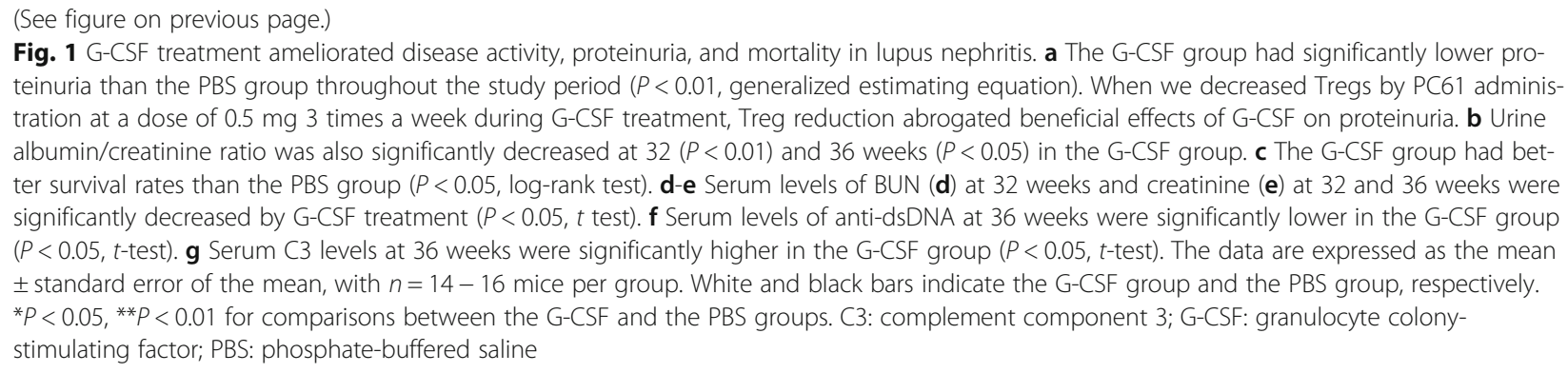

fluorochrome-conjugated isotype-matched irrelevant monoclonal antibodies were used as negative controls. 7-aminoactinomycin D was also added to distinguish between live and dead cells. The stained cells were analyzed using BD FACSCanto II (BD Biosciences).

\section{Statistical analysis}

Data were expressed as the mean \pm standard error of the mean (SEM). Student's $t$-test was used to compare means. Survival rates were analyzed using the Kaplan-Meier method and compared by the log-rank test. Difference in proteinuria during treatment was analyzed using generalized estimating equations. $P$ values of less than 0.05 were considered statistically significant. All data were analyzed using SPSS v.22.0 software (SPSS Inc., Chicago, IL, USA).

\section{Results}

\section{G-CSF treatment ameliorated lupus-like disease in NZB/ W F1 mice}

Proteinuria from semi-quantitative measurement values was around 1.5 in most mice at 24 weeks of age. After 24 weeks, proteinuria gradually increased, reaching up to 3.3 in the PBS group. In contrast, proteinuria was maintained at a low level in the G-CSF group $(P<0.01$, Fig. 1a). Urine albumin/creatinine ratio was significantly decreased at 32 weeks $(P<0.01)$ and 36 weeks $(P<0.05)$ in the GCSF group (Fig. 1b). At 36 weeks of age, $27 \%$ of mice in the PBS group had died, whereas there was no mortality in the G-CSF group $(P<0.05$, Fig. 1c). Serum levels of BUN (Fig. 1d) and creatinine (Fig. 1e) were significantly lower in the G-CSF group compared with the PBS group $(P<0.05)$. G-CSF treatment decreased serum levels of anti-dsDNA IgG $(P<0.05$, Fig. 1f $)$ and increased serum levels of $\mathrm{C} 3(P<0.05$, Fig. 1g).

\section{G-CSF treatment attenuated renal pathologic injury}

Renal injury in NZB/W F1 mice consisted of glomerular, interstitial, and perivascular infiltration of monocytes and lymphocytes, and concomitant immune complex deposition $[21,22]$. NZB/W F1 mice in the PBS group exhibited severe renal injury, which was characterized by glomerulosclerosis, crescent formation, tubular cast deposition, increased mesangial matrix, and diffuse perivascular and interstitial mononuclear cell infiltration. All of these pathological injuries were attenuated in the G-CSF group $(P<0.05$, Fig. 2a). G-CSF treatment also suppressed glomerular deposition of IgG $(P<0.05$, Fig. $2 \mathrm{~b})$ and C3 $(P<$ 0.01, Fig. 2b) in NZB/W F1 mice.

\section{G-CSF treatment suppressed renal infiltration of proinflammatory immune cells and decreased numbers of splenic immune cells}

Renal infiltration of leukocyte subsets was analyzed in NZB/W F1 mice. We found that G-CSF treatment decreased renal infiltration of $\mathrm{CD} 45^{+}$leukocytes $(P<0.01$, Fig. 3a), $\mathrm{CD}^{+}, \mathrm{CD} 4^{+}$and $\mathrm{CD} 8^{+} \mathrm{T}$ cells $(P<0.05$, Fig. $3 \mathrm{~b})$ when compared to that in the PBS group. Renal infiltration of myeloid cells $\left(\mathrm{CD}_{11} \mathrm{~b}^{+}\right)$cells was also decreased in the G-CSF group $(P<0.01$, Fig. 3c). However, numbers of renal $\mathrm{CD} 19^{+} \mathrm{B}$ cells (Fig. $3 \mathrm{~b}$ ), $\mathrm{CD} 11 \mathrm{c}^{+}$dendritic cells (Fig. 3d), and NK cells (NK1.1 ${ }^{+} \mathrm{CD} 3^{-}$, Fig. 3e) were similar between the two groups.

In the spleen, G-CSF treatment decreased the numbers of splenocytes $(P<0.01$, Fig. $4 \mathrm{a})$ including $\mathrm{CD} 19^{+} \mathrm{B}$ cells, $\mathrm{CD}^{+} \mathrm{T}$ cells, $\mathrm{CD} 4^{+} \mathrm{T}$ cells, and $\mathrm{CD} 8^{+} \mathrm{T}$ cells (Fig. $4 \mathrm{~b}$ ). Although there was no difference in the number of splenic $\mathrm{CD}_{11 \mathrm{~b}^{+}}$myeloid cells (Fig. 4c), G-CSF treatment decreased numbers of splenic dendritic cells $(P<0.01$, Fig. 4 d) and NK cells $(P<0.05$, Fig. 4 e), when compared to the PBS group. In addition, the numbers of follicular helper T cells $\left(\mathrm{CD} 4^{+} \mathrm{CXCR} 5^{+} \mathrm{PD}-1^{+}, P<0.05\right.$, Fig. 4f) and plasma cells $\left(\mathrm{CD} 138^{\text {hi }} \mathrm{CD} 19^{\text {low }}, P<0.01\right.$, Fig. $\left.4 \mathrm{~g}\right)$ were also lower in the G-CSF group than in the PBS group.

\section{G-CSF treatment induced expansion of $\mathrm{CD} 4^{+} \mathrm{CD} 25^{+} \mathrm{Foxp}^{+}$ Tregs}

The proportion and absolute numbers of $\mathrm{CD} 4^{+} \mathrm{CD} 25$ ${ }^{+} \mathrm{Foxp}^{+}$Tregs were evaluated in both the kidneys and spleen of NZB/W F1 mice (Fig. 5a-c). G-CSF treatment induced significant expansion of renal $\mathrm{CD} 4{ }^{+} \mathrm{CD} 25$ ${ }^{+}$Foxp $3^{+}$Tregs in both proportion $(P<0.01$, Fig. $5 \mathrm{~b})$ and absolute numbers $(P<0.05$, Fig. $5 b)$. Among CD $4^{+}$Foxp3

${ }^{+} \mathrm{T}$ cells in the kidney, the number of $\mathrm{CD} 4^{+} \mathrm{CD} 25^{-}$Foxp3

+ cells was decreased by G-CSF treatment $(P<0.05$, Fig. 5b). G-CSF treatment also increased the proportion of $\mathrm{CD}^{+} \mathrm{CD} 25^{+} \mathrm{Foxp}^{+}$Tregs in the spleen $(P<0.05$, 


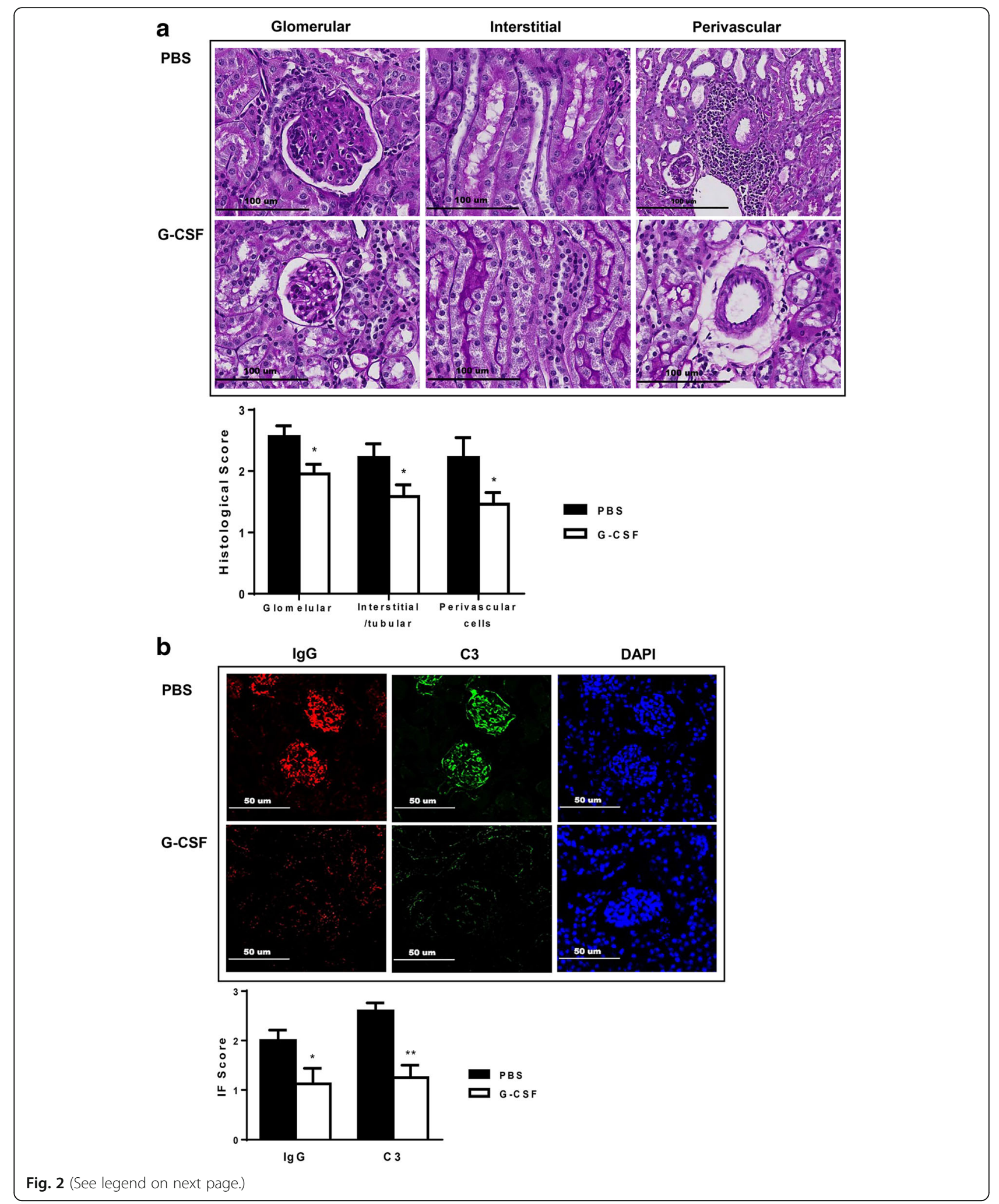


(See figure on previous page.)

Fig. 2 G-CSF treatment ameliorated histologic injury and immune deposits in lupus nephritis. a Representative kidney sections stained with PAS (200x; histological score on the right-panel). b Frozen sections stained for lgG and C3 deposits (200x; immunofluorescence staining score on the right-panel). Scores of both histologic injury and immune deposits were lower in the G-CSF group than the PBS group. Differences in histologic injury and immune deposit scores were analyzed by $t$ tests. The data are expressed as the mean \pm standard error of the mean, with $n=14-16$ mice per group. White and black bars indicate the G-CSF group and the PBS group, respectively. ${ }^{*} P<0.05,{ }^{* *} P<0.01$ for comparisons between the G-CSF and the PBS groups. C3: complement component 3; G-CSF, granulocyte colony-stimulating factor: PAS, periodic acid-Schiff's reagent; PBS: phosphate-buffered saline

Fig. 5c). To assess whether Treg expansion is the main mechanism of G-CSF-mediated renal protection in NZB/W F1 mice, we decreased Tregs by PC61 treatment during G-CSF treatment (Additional file 1) and found that Treg reduction abrogated beneficial effects of GCSF on proteinuria (Fig. 1a).

\section{G-CSF treatment decreased numbers of CD11 $b^{+}$Ly6G} ${ }^{+}$Ly6C ${ }^{\text {low }}$ and CD11 b ${ }^{+}$Ly6G'Ly6C ${ }^{\text {high }}$ myleoid cells Among $\mathrm{CD}_{4} 5^{+} \mathrm{CD}_{11 \mathrm{~b}^{+}}$myeloid cells, Ly6G Ly6C ${ }^{\text {high }}$ cells are considered to be inflammatory monocytes or monocytic MDSCs, and $\mathrm{Ly}_{6 \mathrm{G}}{ }^{+} \mathrm{Ly} 6 \mathrm{C}^{\mathrm{low}}$ cells are inflammatory granulocytes or granulocytic MDSCs. In spleen, G-CSF treatment decreased proportions and absolute numbers of both Ly6G ${ }^{+}$Ly6C ${ }^{\text {low }}$ and Ly6G Ly6C ${ }^{\text {high }}$ cells (Fig. 6a). Furthermore, G-CSF treatment decreased renal infiltration of Ly6G ${ }^{+}$Ly6 $C^{\text {low }}$ and Ly6G ${ }^{-}$Ly6C $C^{\text {high }}$ cells; however, impact on Ly6G ${ }^{+}$Ly6 $\mathrm{C}^{\text {low }}$ was not statistically significant (Fig. 6b).

\section{G-CSF treatment suppressed expression of} proinflammatory cytokines in NZB/W F1 mice

G-CSF treatment decreased levels of MCP-1, IL-6, and TNF- $\alpha$ in either serum (Fig. 7a) or renal tissues (Fig. 7b). G-CSF treatment also decreased expression of IL-2, IL10 , and IL-17, which are important type 1 helper T cells (Th1), type 2 helper $\mathrm{T}$ cells (Th2), and type 17 helper $\mathrm{T}$ cells (Th17) cytokines, respectively. Overall, G-CSF treatment decreased expression of proinflammatory chemokine and cytokines in renal tissues as well as systemically in NZB/W F1 mice.

\section{Low-dose G-CSF treatment did not expand regulatory $\mathrm{T}$ cells or ameliorate lupus nephritis}

When we administered $250 \mu \mathrm{g} / \mathrm{kg}$ of G-CSF 3 times a week, G-CSF did not attenuate proteinuria (Additional file 2a) or mortality (Additional file 2b). There was no significant difference in either anti-ds DNA levels (Additional
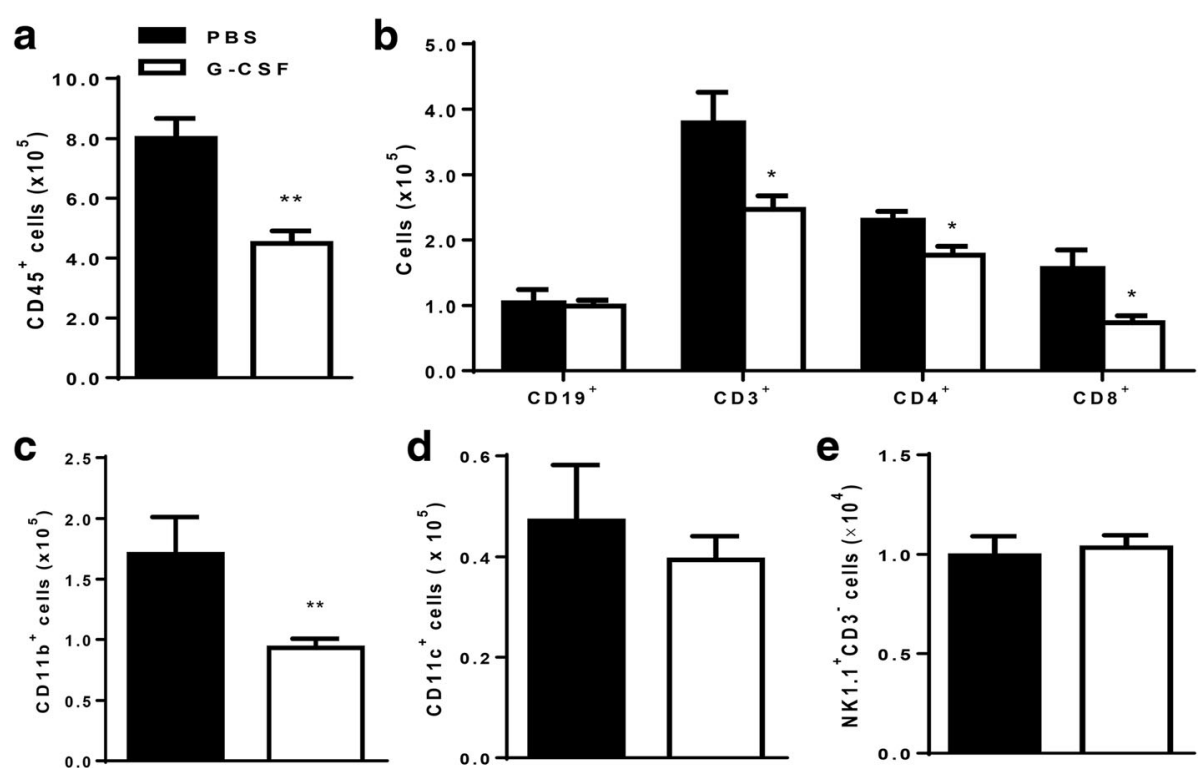

Fig. 3 G-CSF treatment attenuated renal infiltration of inflammatory leukocytes. a Absolute number of total leukocytes $\left(C D 45^{+}\right)$in kidney was significantly decreased in the G-CSF group $(P<0.01)$. b Number of infiltrating T cells $\left(C D 3^{+}\right)$including both $C D 4^{+}$and $C D 8^{+} T$ cells was decreased in the G-CSF group $(P<0.05)$, whereas number of infiltrating B cells $\left(C D 19^{+}\right)$was not decreased. $\mathbf{c}$ Numbers of $C D 11 b^{+}$myeloid cells were lower in the G-CSF group than in the PBS group $(P<0.01)$. d-e However, G-CSF did not decrease renal infiltration of either CD11 $c^{+}$dendritic cells $(\mathbf{d})$ or NK cells $\left(\mathrm{NK}_{1.1} 1^{+} \mathrm{CD} 3^{-}\right.$, e). Flowcytometric analysis was used for quantifying renal cell types. The data are expressed as the mean \pm standard error of the mean, with $n=4-5$ mice per group. White and black bars indicate the G-CSF group and the PBS group, respectively. ${ }^{*} P<0.05$, ${ }^{* *} P<0.01$ for comparisons between the G-CSF and the PBS groups using t-test. G-CSF: granulocyte colony-stimulating factor; PBS: phosphate-buffered saline 

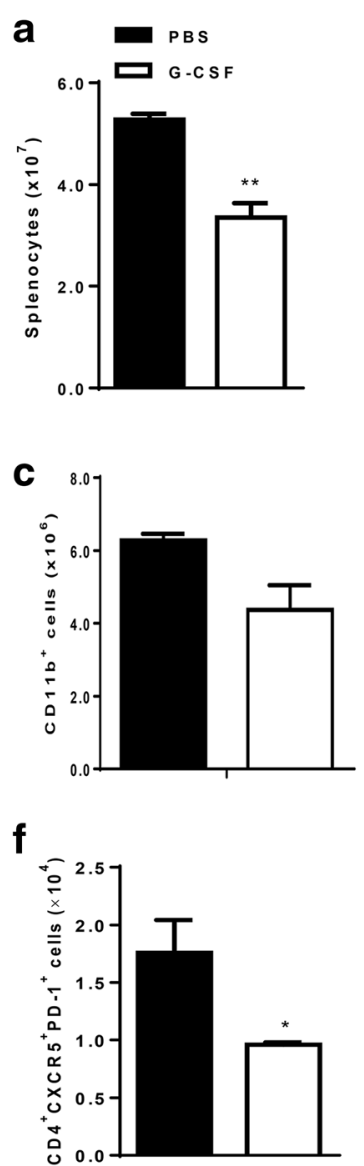
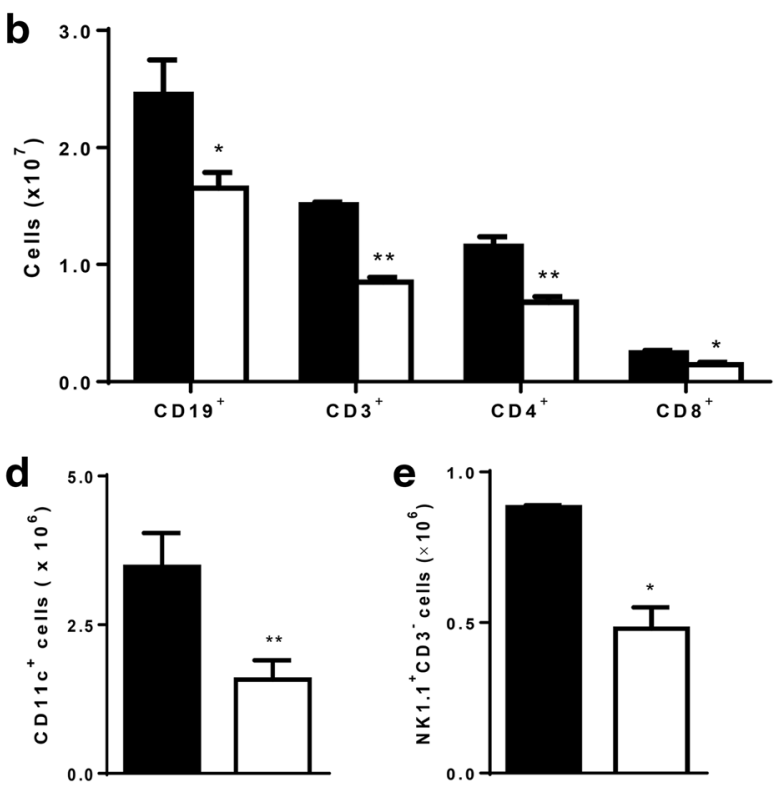

g

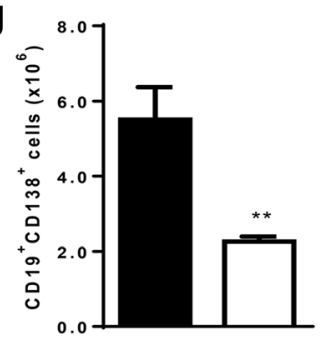

Fig. 4 G-CSF treatment decreased numbers of splenic immune cells. a Absolute number of total splenocytes was lower in the G-CSF group $(P<0.01)$. $\mathbf{b}$ Numbers of B cell $\left(C D 19^{+}\right)$, T cells $\left(\mathrm{CD}^{+}\right)$, and T cell subsets $\left(C D 4^{+}, \mathrm{CD}^{+}\right)$were decreased by G-CSF treatment. $\mathbf{c}$-e Number of CD11 b ${ }^{+}$myeloid cells $(\mathbf{c})$ was not significantly decreased by G-CSF, whereas numbers of CD1 1 $\mathrm{c}^{+}$dendritic cells (d) and NK cells (NK1.1 $\left.1^{+} \mathrm{CD} 3^{-}, \mathbf{e}\right)$ were significantly lower in the G-CSF group. $\mathbf{f}-\mathbf{g}$ Numbers of both follicular helper T cells (CD4 ${ }^{+}$CXCR5 ${ }^{+}$PD- $1^{+}$, $\mathbf{f}$ ) and plasma cells $\left(C D 19^{\text {low }}\right.$ CD $138^{\text {hi }}$, $\left.\mathbf{g}\right)$ were lower in the G-CSF group. Flowcytometric analysis was used for quantifying spleen cell types. The data are expressed as the mean \pm standard error of the mean, with $n=4-5$ mice per group. White and black bars indicate the G-CSF group and the PBS group, respectively. ${ }^{*} P<0.05$, ${ }^{* *} P<0.01$ for comparisons between the G-CSF and the PBS groups using t-test. G-CSF: granulocyte colony-stimulating factor; PBS: phosphatebuffered saline

file 2c) or serum C3 levels (Additional file 2d). Histologic injury was also not improved (Additional file 2e). In parallel, Tregs were not expanded in kidneys (Additional file 2f) or spleen (Additional file 2g). Low-dose GCF treatment did not decrease inflammatory granulocytes (Additional file $2 \mathrm{~h}-\mathrm{i}$ )."

\section{Discussion}

This study demonstrated that G-CSF treatment decreased proteinuria, serum levels of BUN and creatinine, and ultimately decreased mortality of NZB/W F1 mice through the expansion of Tregs. G-CSF treatment also decreased serum levels of anti-dsDNA, increased serum levels of $\mathrm{C} 3$, and attenuated renal tissue injury including deposition of IgG and C3 in NZB/W F1 mice. Furthermore, GCSF treatment induced expansion of $\mathrm{CD} 44^{+} \mathrm{CD} 25^{+} \mathrm{Foxp} 3^{+}$ Tregs, with decreased renal infiltration of $\mathrm{T}$ cells, $\mathrm{B}$ cells, inflammatory granulocytes and monocytes rather than MDSCs. G-CSF treatment also decreased expression of MCP-1, IL-6, TNF- $\alpha$, IL-2, IL-10, and IL-17.

Chronic treatment of low-dose G-CSF $(10 \mu \mathrm{g} / \mathrm{kg})$ aggravated lupus nephritis in MRL/lpr mice, where these results might be attributed to increased Th2 cytokines and IFN- $\alpha[3,7,13]$. Conversely, chronic treatment of high-dose G-CSF $(200 \mu \mathrm{g} / \mathrm{kg})$ attenuated lupus nephritis in MRL/lpr mice by potentially decreasing glomerular expression of FCRyIII and IL-12 production [13]. The GCSF dose in this study was comparable to the high-dose of G-CSF in the previous study [13]. When we reduced GCSF dose by $40 \%$, we could not ameliorate lupus nephritis. Proinflammatory chemokines (MCP-1) and cytokines (IL-6, TNF- $\alpha$ ), Th1 cytokines (IL-2, IFN- $\gamma$ ), Th2 cytokines (IL-4, IL-10), and Th17 cytokines (IL-17A) are involved in pathogenesis of lupus nephritis, and are therapeutic 


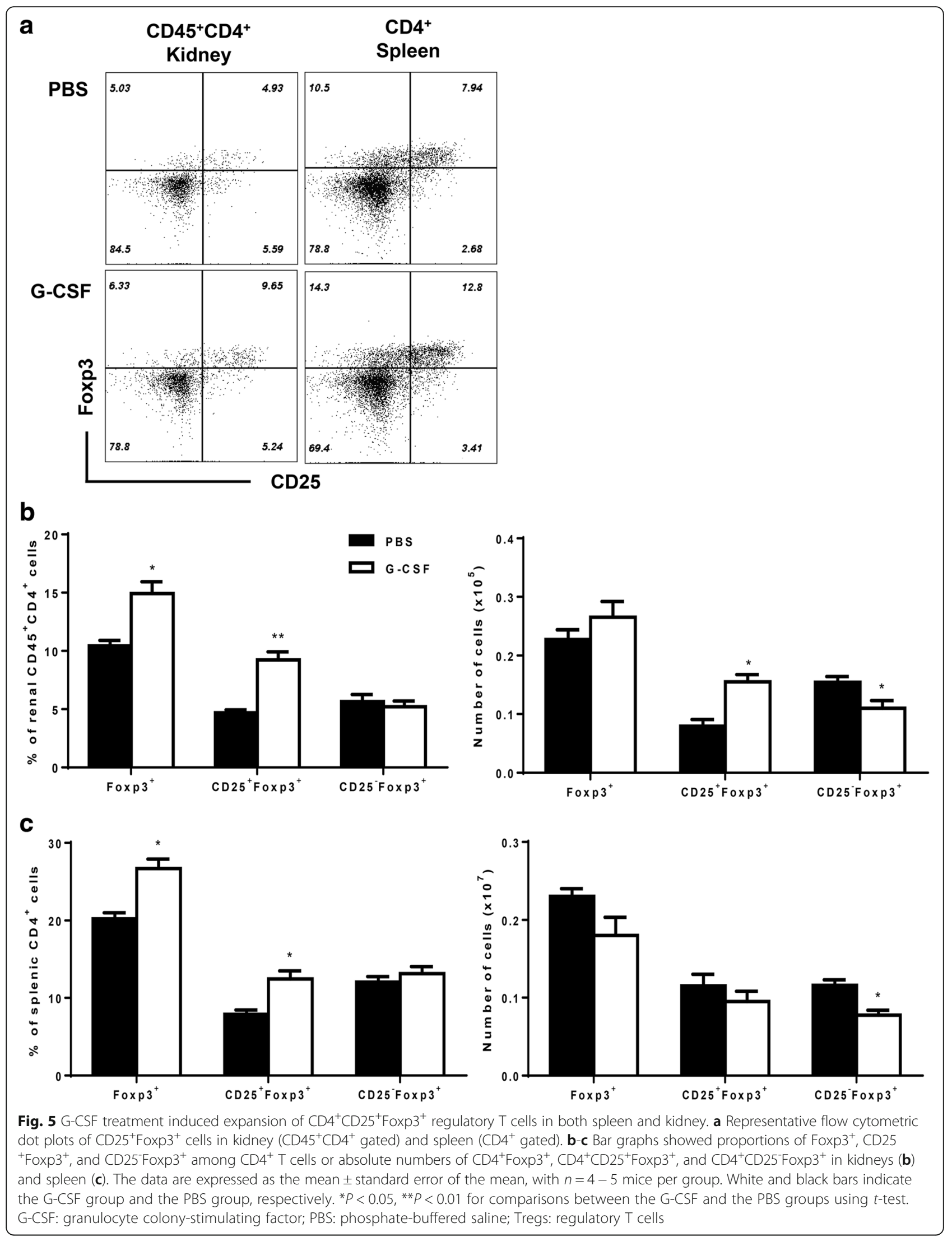


a
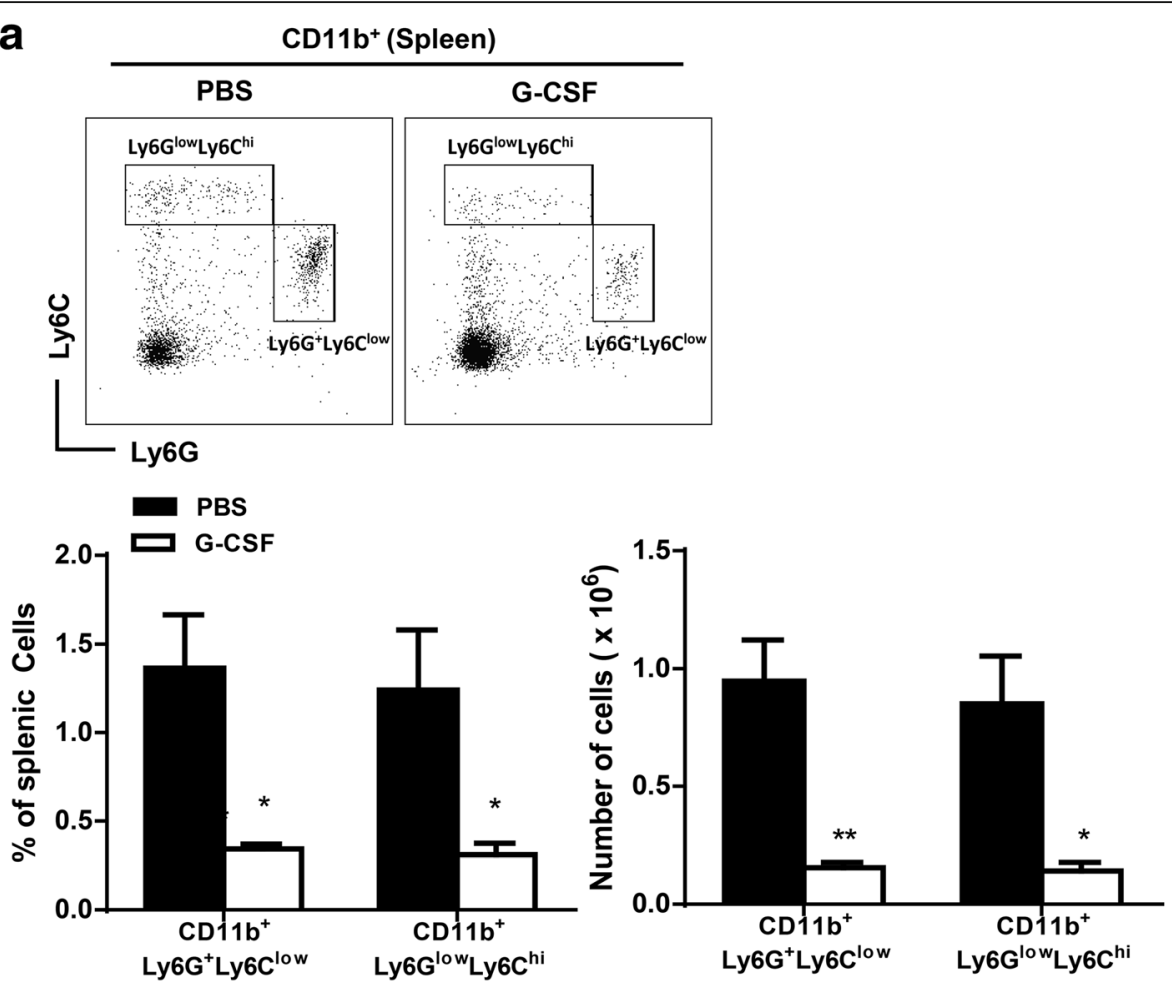

b
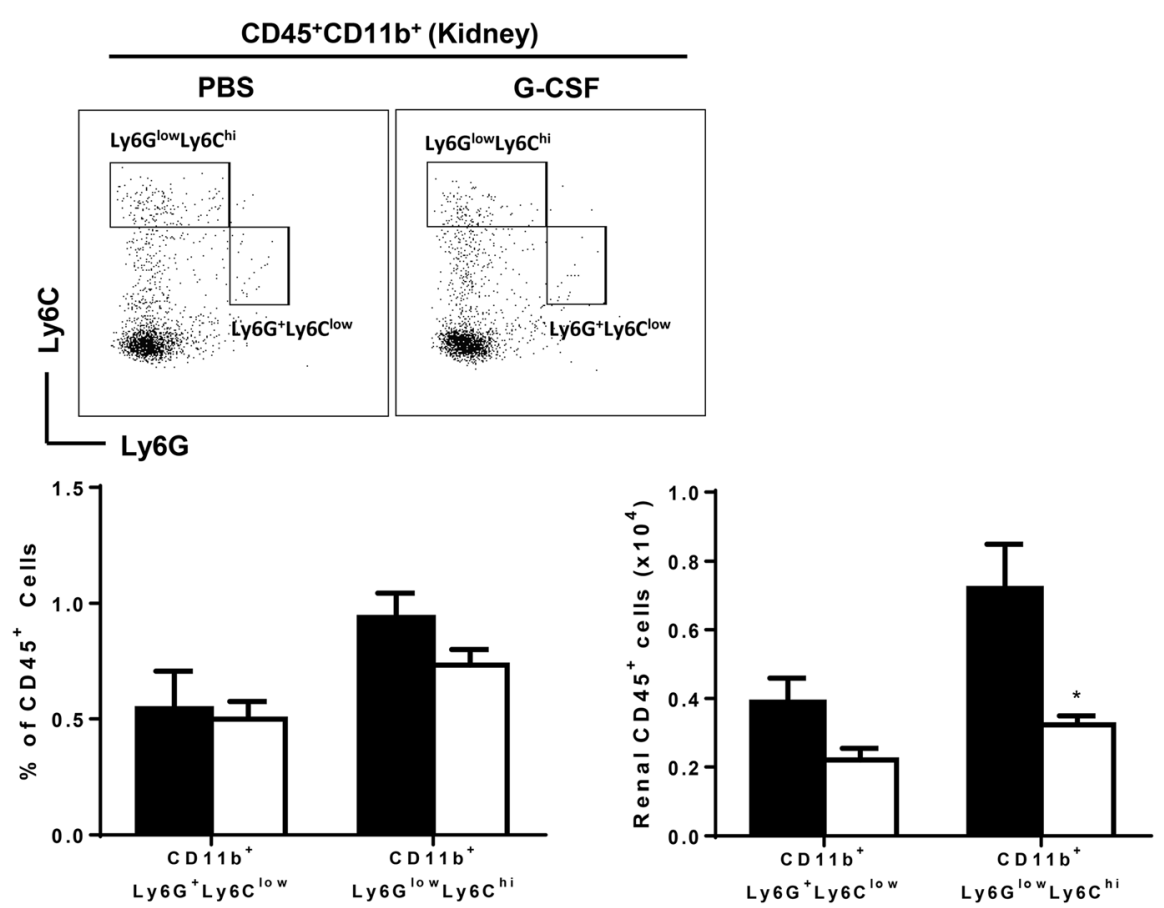

Fig. 6 (See legend on next page.) 
(See figure on previous page.)

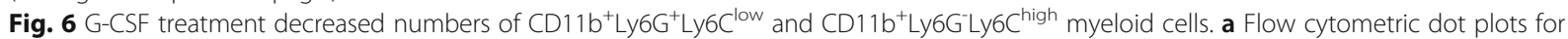
Ly6G and Ly6C defined CD11 b+ Ly6G ${ }^{+}$Ly6C $C^{\text {low }}$ and CD11 b+ Ly6G Ly6Chigh cells in spleen (CD11 b ${ }^{+}$gated). Bar graphs on the left side showed proportions of $C D 11 b^{+} L_{6} 6 G^{+} \mathrm{Ly} 6 C^{l o w}$ and $C D 11 b^{+} L y 6 G{ }^{-} L y 6 C^{\text {high }}$ cells among total splenocytes. Bar graphs on the right side indicated absolute numbers of $C D 11 b^{+} L_{6} 6 G^{+}$Ly6 $C^{\text {low }}$ and $C D 11 b^{+}$Ly6G Ly6C high cells in spleen. b Flow cytometric dot plots for Ly6G and Ly6C defined CD $11 b^{+} L y 6 G$ ${ }^{+}$Ly6 $C^{\text {low }}$ and $C D 11 b^{+}$Ly6G Ly6C ${ }^{\text {high }}$ cells in kidney $\left(C D 45^{+} C D 11 b^{+}\right.$gated). Bar graphs on the left side showed proportions of CD11 $b^{+} L y 6 G$ ${ }^{+}$Ly6C ${ }^{\text {low }}$ and $C D 11 b^{+}$Ly6G Ly6C high cells among renal CD45 cells. Bar graphs on the right side indicated absolute numbers of CD $11 b^{+} L y 6 G$ ${ }^{+}$Ly6C ${ }^{\text {low }}$ and $C D 11 b^{+}$Ly6G Ly6C $C^{\text {high }}$ cells in kidney. The data are expressed as the mean \pm standard error of the mean, with $n=4-5$ mice per group. White and black bars indicate the G-CSF group and the PBS group, respectively. ${ }^{*} P<0.05,{ }^{*} P<0.01$ for comparisons between the G-CSF and the PBS groups using t-test. G-CSF: granulocyte colony-stimulating factor; PBS: phosphate-buffered saline; Tregs: regulatory T cells

targets for lupus nephritis [23, 24]. G-CSF treatment decreased the levels of these pathogenic chemokine/cytokines such as MCP-1, IL-6, TNF- $\alpha$, IL-2, IL-10, and IL-17, and thus were consistent with its suppressive effects on both innate and adaptive immune cells in NZB/W F1 mice. Despite reduced IL-10 levels, reduced levels of IL-6 in both serum and renal tissues might have contributed towards Treg expansion by G-CSF treatment. Taken together, these results indicated that the suppression of various proinflammatory cytokines involved in lupus nephritis

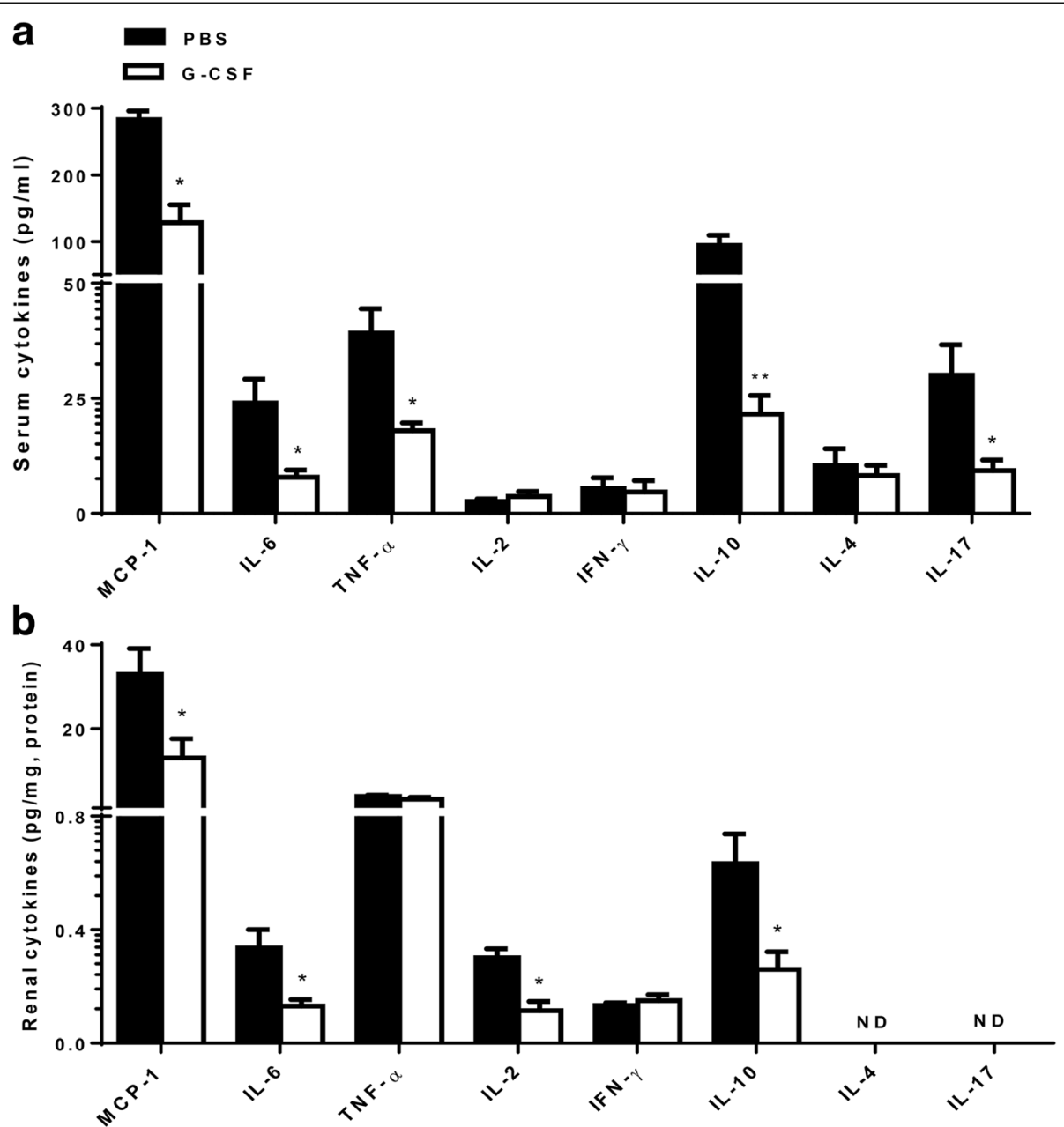

Fig. 7 G-CSF treatment decreased levels of proinflammatory chemokine and cytokines in the serum and kidneys. Expression levels of proinflammatory chemokine (MCP-1), proinflammatory cytokines (IL-6, TNF-a), Th1 cytokines (IL-2, INF- $\mathrm{f})$, Th2 cytokines (IL-4, IL-10), and Th17 cytokines (IL-17A) were measured in serum (a) and renal tissues (b). G-CSF treatment significantly decreased serum levels of MCP-1, IL-6, TNF-a, IL-10 and IL-17. In renal tissues, G-CSF treatment significantly decreased levels of MCP-1, IL-6, IL-2, and IL-10. The data are expressed as the mean \pm standard error of the mean, with $n=4-7$ mice per group. White and black bars indicate the G-CSF group and the PBS group, respectively. ${ }^{*} P<$ $0.05,{ }^{*} P<0.01$ for comparisons between the G-CSF and the PBS groups using t-test. G-CSF: granulocyte colony-stimulating factor; IFN: interferon; IL: interleukin; MCP-1: monocyte chemoattractant protein-1; PBS: phosphate-buffered saline; Th: helper T cells; TNF: tumor necrosis factor; Tregs: regulatory $T$ cells 
pathogenesis could have led to the G-CSF-mediated attenuation of lupus nephritis.

G-CSF decreased inflammatory granulocytes (CD11b

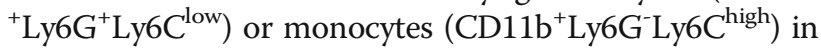
both kidney and spleen. These results were consistent with previous studies in murine lupus models. In those studies, the percentage of $\mathrm{CD} 11 \mathrm{~b}^{+} \mathrm{Gr}-1^{\text {low }}$ monocytes in the kidney increased as the disease progressed in the MRL/lpr mouse model of lupus, and it expanded in B6 mice with established chronic graft-versus-host disease $[12,25]$. Although inflammatory granulocytes and monocytes share surface markers with the MDSCs, these data suggested that G-CSF treatment decreased inflammatory granulocytes and monocytes rather than MDSCs in parallel with improvement of lupus nephritis.

We found that Tregs expanded in the G-CSF group and Treg depletion abrogated the G-CSF-mediated beneficial effects on proteinuria in NZB/W F1 mice. These findings were consistent with a previous study, which demonstrated that G-CSF recruited Tregs, and thereby prevented onset of spontaneous diabetes in NOD mice [26]. $\mathrm{CD} 4^{+} \mathrm{CD} 25^{+}$Treg cells were decreased in SLE patients and Treg cell numbers were inversely correlated with disease activity [27, 28]. Moreover, infusion of Tregs attenuated disease progression and reduced mortality in murine lupus [29]. Therefore, we assumed that Treg expansion could be the beneficial mechanism of G-CSF on lupus nephritis in this study. Although MDSC could contribute to induction of Tregs [30], lack of MSDC expansion might exclude this mechanism in our setting. It was also observed that G-CSF decreased CD25Foxp $3^{+} \mathrm{T}$ cells in contrast to $\mathrm{CD} 25^{+} \mathrm{Foxp}^{+}{ }^{+}$Tregs. These results demonstrated another benefit of G-CSF treatment for lupus nephritis, because CD25 ${ }^{-} \mathrm{Foxp}^{+}{ }^{+} \mathrm{T}$ cells were increased in the case of active lupus nephritis and had lower suppressive activity than CD25 ${ }^{+}$Foxp $3^{+}$Tregs $[31,32]$.

Beneficial effects of G-CSF for lupus nephritis observed in this study suggest G-CSF as potential treatment for lupus nephritis, considering G-CSF is already used in clinical treatment of neutropenia after chemotherapy. Human G-CSF is less potent in mice than murine G-CSF, thus we used a very high dose of human GCSF in NZB/W F1 mice. We expect to reduce this dose to tolerable levels in clinical trials.

Most patients with lupus nephritis take long-term immunosuppressants such as corticosteroids, cyclophosphamide, or mycophenolate mofetil. Therefore, one of the limitations of this study is that we did not evaluate the interacting effects of G-CSF with standard immuosuppressants for lupus nephritis. Further studies are needed to confirm our findings in clinically plausible situations.

\section{Conclusions}

G-CSF treatment ameliorated lupus nephritis, and preferential expansion of $\mathrm{CD} 4^{+} \mathrm{CD} 25^{+} \mathrm{Foxp}^{+}$Tregs instead of
MDSC may be the underlying mechanism. Therefore, GCSF may have a therapeutic potential for lupus nephritis.

\section{Additional files}

Additional file 1: PC61 treatment markedly decreased regulatory $T$ cells in both kidneys and spleen. During G-CSF treatment, we administered anti-CD25 depleting antibodies (PC61) at a dose of $0.5 \mathrm{mg} 3$ times a week from 33 weeks to 36 weeks. At harvest, regulatory T cells were depleted in spleen and markedly decreased in kidneys in the G-CSF plus PC61 group. (TIF 4173 kb)

Additional file 2: Low-dose G-CSF treatment did not expand regulatory $T$ cells or ameliorate lupus nephritis. When we administered $250 \mu \mathrm{g} / \mathrm{kg}$ of G-CSF 3 times a week, G-CSF did not attenuate proteinuria (a) or mortality (b). There was no significant difference in either anti-ds DNA levels (c) or serum C3 levels (d). Histologic injury was also not improved (e). In parallel, Tregs were not expanded in kidneys (f) or spleen (g). Low-dose GCF treatment did not decrease inflammatory granulocytes or monocytes in kidney (h) or spleen (i). (TIF 2014 kb)

\section{Abbreviations}

Anti-dsDNA: Anti-double stranded DNA; C3: Complement 3; GCSF: Granulocyte colony-stimulating factor; IFN: Interferon; IL: Interleukin; MDSC: Myeloid-derived suppressor cells; PAS: Periodic acid-Schiff's; PBS: Phosphate-buffered saline; SLE: Systemic lupus erythematosus; TNF: Tumor necrosis factor; Tregs: Regulatory $T$ cells

\section{Acknowledgement}

We would like to thank Kyowa Kirin for the generous supply of G-CSF.

\section{Funding}

This study was supported by a grant from the Korean Health Technology R\&D Project, Ministry of Health \& Welfare, Republic of Korea (project no. A120641).

\section{Availability of data and materials}

All data underlying the findings are presented in the main paper and its Additional files 1 and 2 .

\section{Authors' contributions}

J-JY, EJ, CA, and JY designed research. J-JY, EJ, J-GL, JYJ, JMS, MH and TYK performed research. J-JY, EJ and JY wrote manuscript, and CA reviewed manuscript critically. All authors read and approved the final manuscript.

\section{Competing interests}

The authors declared no potential conflict of interests with respect to the research, authorship, and/or publication of this article.

\section{Consent for publication}

Not applicable.

\section{Ethics approval}

Animal experiments were approved by the Committee on Animal Care and Use at Seoul National University College of Medicine.

\section{Author details}

${ }^{1}$ Transplantation Research Institute, Seoul National University College of Medicine, Seoul 03080, Republic of Korea. ${ }^{2}$ Department of Internal Medicine, Seoul National University College of Medicine, Seoul 03080, Republic of Korea. ${ }^{3}$ Transplantation Center, Seoul National University Hospital, Seoul 03080, Republic of Korea. ${ }^{4}$ Department of Surgery, Seoul National University Hospital, Seoul 03080, Republic of Korea.

Received: 19 March 2016 Accepted: 26 October 2016

Published online: 15 November 2016

\section{References}

1. Mok CC, Lau CS. Pathogenesis of systemic lupus erythematosus. J Clin Pathol. 2003:56(7):481-90.

2. Tsokos GC. Systemic lupus erythematosus. N Engl J Med. 2011;365(22):2110-21. 
3. Rutella S. Granulocyte colony-stimulating factor for the induction of T-cell tolerance. Transplantation. 2007;84(1 Suppl):S26-30

4. Rutella S, Pierelli L, Bonanno G, Sica S, Ameglio F, Capoluongo E, et al. Role for granulocyte colony-stimulating factor in the generation of human $T$ regulatory type 1 cells. Blood. 2002;100(7):2562-71.

5. Zou L, Barnett B, Safah H, Larussa VF, Evdemon-Hogan M, Mottram P, et al. Bone marrow is a reservoir for CD4 + CD25+ regulatory $T$ cells that traffic through CXCL12/CXCR4 signals. Cancer Res. 2004;64(22):8451-5.

6. Arpinati M, Green CL, Heimfeld S, Heuser JE, Anasetti C. Granulocyte-colony stimulating factor mobilizes T helper 2-inducing dendritic cells. Blood. 2000; 95(8):2484-90.

7. Rutella S, Bonanno G, Pierelli L, Mariotti A, Capoluongo E, Contemi AM, et al. Granulocyte colony-stimulating factor promotes the generation of regulatory DC through induction of IL-10 and IFN-alpha. Eur J Immunol. 2004;34(5):1291-302

8. Hartung T, Docke WD, Gantner F, Krieger G, Sauer A, Stevens P, et al. Effect of granulocyte colony-stimulating factor treatment on ex vivo blood cytokine response in human volunteers. Blood. 1995;85(9):2482-9.

9. Adeegbe D, Serafini P, Bronte V, Zoso A, Ricordi C, Inverardi L. In vivo induction of myeloid suppressor cells and CD4(+)Foxp3(+) T regulatory cells prolongs skin allograft survival in mice. Cell Transplant. 2011;20(6):941-54.

10. Lees JR, Azimzadeh AM, Bromberg JS. Myeloid derived suppressor cells in transplantation. Curr Opin Immunol. 2011;23(5):692-7.

11. Cripps JG, Gorham JD. MDSC in autoimmunity. Int Immunopharmacol. 2011; 11(7):789-93.

12. Lantow M, Sivakumar R, Zeumer L, Wasserfall C, Zheng YY, Atkinson MA, et al. The granulocyte colony stimulating factor pathway regulates autoantibody production in a murine induced model of systemic lupus erythematosus. Arthritis Res Ther. 2013;15(2):R49.

13. Zavala F, Masson A, Hadaya K, Ezine S, Schneider E, Babin O, et al. Granulocyte-colony stimulating factor treatment of lupus autoimmune disease in MRL-Ipr/lpr mice. J Immunol. 1999;163(9):5125-32.

14. Vasiliu IM, Petri MA, Baer AN. Therapy with granulocyte colony-stimulating factor in systemic lupus erythematosus may be associated with severe flares. J Rheumatol. 2006;33(9):1878-80.

15. Sakai T, Hatano Y, Abe I, Ishii K, Fujiwara S. A case of an SLE patient with febrile neutropenia who experienced exacerbation of cutaneous manifestations after the administration of G-CSF. Mod Rheumatol. 2013; 23(6):1231-6.

16. Theofilopoulos AN, Dixon FJ. Murine models of systemic lupus erythematosus. Adv Immunol. 1985;37:269-390.

17. Stanic AK, Stein CM, Morgan AC, Fazio S, Linton MF, Wakeland EK, et al. Immune dysregulation accelerates atherosclerosis and modulates plaque composition in systemic lupus erythematosus. Proc Natl Acad Sci U S A. 2006;103(18):7018-23.

18. Menke J, Bork T, Kutska B, Byrne KT, Blanfeld M, Relle M, et al. Targeting transcription factor Stat4 uncovers a role for interleukin-18 in the pathogenesis of severe lupus nephritis in mice. Kidney Int. 2011;79(4):452-63.

19. Stirzaker RA, Biswas PS, Gupta S, Song L, Bhagat G, Pernis AB. Administration of fasudil, a ROCK inhibitor, attenuates disease in lupus-prone NZB/W F1 female mice. Lupus. 2012;21(6):656-61.

20. Lee SW, Park YB, Yang J, Park KH, Lee SK, Choi KH, et al. Attenuation of nephritis in lupus-prone mice by thalidomide. Rheumatology (Oxford). 2012; 51(12):2131-40.

21. Hurd ER, Ziff M. Quantitative studies of immunoglobulin deposition in the kidney, glomerular cell proliferation and glomerulosclerosis in NZB/NZW F1 hybrid mice. Clin Exp Immunol. 1977;27(2):261-8.

22. Zurier RB, Damjanov I, Sayadoff DM, Rothfield NF. Prostaglandin E1 treatment of NZB/NZW F1 hybrid mice. II Prevention of glomerulonephritis. Arthritis Rheum. 1977;20(8):1449-56.

23. Lech M, Anders HJ. The pathogenesis of lupus nephritis. J Am Soc Nephrol. 2013;24(9):1357-66.

24. Gottschalk TA, Tsantikos E, Hibbs ML. Pathogenic inflammation and its therapeutic targeting in systemic lupus erythematosus. Front Immunol. 2015;6:550.

25. Iwata Y, Furuichi K, Kitagawa K, Hara A, Okumura T, Kokubo S, et al. Involvement of CD11b + GR-1 low cells in autoimmune disorder in MRL-Fas Ipr mouse. Clin Exp Nephrol. 2010;14(5):411-7.

26. Kared H, Masson A, Adle-Biassette H, Bach JF, Chatenoud L, Zavala F. Treatment with granulocyte colony-stimulating factor prevents diabetes in
NOD mice by recruiting plasmacytoid dendritic cells and functional CD4(+ )CD25(+) regulatory T-cells. Diabetes. 2005;54(1):78-84.

27. Crispin JC, Martinez A, Alcocer-Varela J. Quantification of regulatory T cells in patients with systemic lupus erythematosus. J Autoimmun. 2003:21(3):273-6.

28. Lyssuk EY, Torgashina AV, Soloviev SK, Nassonov EL, Bykovskaia SN. Reduced number and function of CD4 + CD25highFoxP3+ regulatory T cells in patients with systemic lupus erythematosus. Adv Exp Med Biol. 2007;601:113-9.

29. Weigert O, von Spee C, Undeutsch R, Kloke L, Humrich JY, Riemekasten G. CD4 + Foxp3+ regulatory T cells prolong drug-induced disease remission in (NZBxNZW) F1 lupus mice. Arthritis Res Ther. 2013;15(1):R35.

30. Huang B, Pan PY, Li Q, Sato Al, Levy DE, Bromberg J, et al. Gr-1 + CD115+ immature myeloid suppressor cells mediate the development of tumorinduced T regulatory cells and T-cell anergy in tumor-bearing host. Cancer Res. 2006;66(2):1123-31.

31. Bonelli M, Goschl L, Bluml S, Karonitsch T, Steiner CW, Steiner G, et al. CD4(+ CD25(-)Foxp3(+) T cells: a marker for lupus nephritis? Arthritis Res Ther. 2014;16(2):R104.

32. Zhang B, Zhang X, Tang FL, Zhu LP, Liu Y, Lipsky PE. Clinical significance of increased CD4 + CD25-Foxp3+ T cells in patients with new-onset systemic lupus erythematosus. Ann Rheum Dis. 2008;67(7):1037-40.

\section{Submit your next manuscript to BioMed Central and we will help you at every step:}

- We accept pre-submission inquiries

- Our selector tool helps you to find the most relevant journal

- We provide round the clock customer support

- Convenient online submission

- Thorough peer review

- Inclusion in PubMed and all major indexing services

- Maximum visibility for your research

Submit your manuscript at www.biomedcentral.com/submit
C) Biomed Central 